\title{
Stochastic ship roll motion via path integral method
}

\author{
G. Cottone ${ }^{1}$, M. Di Paola ${ }^{1}$, R. Ibrahim ${ }^{2}$, A. Pirrotta ${ }^{1}$ R. Santoro ${ }^{1}$ \\ ${ }^{I}$ Dipartimento di Ingegneria Strutturale Aerospazialee Geotecnica, Viale delle Scienze, Palermo, Italy. \\ ${ }^{2}$ Wayne State University, Department of Mechanical Engineering, Detroit, USA.
}

\begin{abstract}
The response of ship roll oscillation under random ice impulsive loads modeled by Poisson arrival process is very important in studying the safety of ships navigation in cold regions. Under both external and parametric random excitations the evolution of the probability density function of roll motion is evaluated using the path integral (PI) approach. The PI method relies on the Chapman-Kolmogorov equation, which governs the response transition probability density functions at two close intervals of time. Once the response probability density function at an early close time is specified, its value at later close time can be evaluated. The PI method is first demonstrated via simple dynamical models and then applied for ship roll dynamics under random impulsive white noise excitation.
\end{abstract}

KEY WORDS: Ship roll; Random impulsive ice loading; Poisson distribution; Path integral; Parametric random excitation; Chapman-Kolmogorov equation.

\section{INTRODUCTION}

The influence of floating ice on the dynamic behavior of ships and offshore structures depends on many factors such as ice thickness and its relative speed with respect to the floating structure. The ice resistance to ship motion forms an essential problem in ship design and navigation. Furthermore, local or global ice loads acting on ocean systems are random and non-smooth when impact interaction takes place. Impact loads on the bow of a ship navigating in solid ice may be modeled by a Poisson law. The assessment of ice related problems encountered by offshore structures as well as by ships during their navigation has recently been documented by Ibrahim et al. (2007). In view of ice loads on marine systems, new design regulations have been introduced by international organizations that are involved in the design and building of ships as well as offshore structures.

Ice loads acting on ocean systems are random in nature and have non-smooth characteristics when they are of impact type. In full-scale experiments, measurements of ice local and global loads revealed randomness in the ice forces and pressures (see, e.g., Meyerhoff and Schlachter, 1980; and Timco and Johnston, 2004). In some cases, ice loads are of impact type and have been assumed as a Poisson arrival process of loading events. Thus, one must deal with probabilistic approaches when studying ships' stochastic stability, response, and reliability. The treatment of dynamical systems under Poisson random processes has been considered in references (e.g., Köylüoğlu, et al., 1995; Di

Corresponding author: Raouf A. Ibrahim

E-mail:ibrahim@eng.wayne.edu
Paola and Pirrotta, 1999; and Proppe, 2003).

For systems under normal or non-normal white noise, the response statistics may be obtained by solving the FokkerPlank Kolmogorov (FPK) equation or the Kolmogorov-Feller equation. However, exact solutions of the partial differential equations governing the evolution of the response probability density function $(p d f)$ are known only for very few cases as shown by Caughey and Dienes (1961), Dimentberg (1982) and Vasta, 1995). Alternatively, several approximate solutions techniques have been developed including variational methods based on eigenfunction expansion of the transition $p d f$ (Atkinson, 1973), finite element method (Spencer and Bergman, 1993) and the path integration approach (Köylüoğlu et al., 1995). The PI approach is an effective tool for evaluating the response in terms of probability density at each time instant, for evaluating moments of various orders, energy response $p d f$, first passage time of strong nonlinear systems. This approach is based on rewriting the FPK equation in integral form in which the kernel is the transition probability density function. Thus one can evaluate the response $p d f$ at time $(t+\tau)$ when its value at an early close time instant $(t)$ is already specified. The crucial point is to define the kernel according to the system under investigation. In the case of normal white noise, if $\tau$ is small, even if the system is nonlinear, the transition $p d f$ is almost Gaussian (short-time Gaussian approximation). It follows that the kernel of the integral form is Gaussian and this simplifies the analysis as shown by Barone et al. (2008). The accuracy of this method was validated using Monte Carlo simulation and the exact solution when the latter is available. Recently, Di Paola and Santoro (2008) have studied the case of Poisson white noise by evaluating the conditional 
probability density function ( $c p d f)$ in order to apply the PI method also for these systems.

The path integral technique was applied to the roll nonlinear motion of a ship in irregular waves by Kwon et al (1993). The exciting moment due to the irregular waves was modeled as a non-white noise. Both damping and nonlinear restoring functions were included with the equivalent whitenoise intensity. Lin and Yim (1995) developed a stochastic analysis scheme to examine the properties of chaotic roll motion and capsize of ships subjected to periodic excitation with a random noise disturbance. The associated FokkerPlanck equation governing the evolution of the probability density function of the roll motion was numerically solved by the path integral solution procedure to obtain joint probability density functions in state space. It was found that the presence of noise enlarges the boundary of the chaotic domains and bridges coexisting attracting basins in the local regimes. The probability of capsize was considered as an extreme excursion problem with the time-averaged probability density function as an invariant measure. Another version of the path integration approach based on the GaussLegendre quadrature integration rule was proposed by $\mathrm{Gu}$ (2006). It was applied for estimating the probability density of the nonlinear roll motion of ships in stochastic beam seas. The ship roll motion was described by a nonlinear random differential equation that includes a nonlinear damping moment and restoring moment. The results include the timeevolution of the ship response probability density as well as the tail region, where the probability value is very important for the system reliability analysis.

The case of small ships with water on deck subjected to random beam waves described by to a periodic force and white noise perturbation was considered by Liqin and Yougang (2007) using the path integral solution. This type of ship motion is governed by two dynamical regions: homoclinic and heteroclinic, where the heteroclinic model emulates symmetric vessel capsize and the homoclinic model represents a vessel with an initial bias caused by water on deck. The random Melnikov mean square criterion was used to determine the parameter domain for the ship's stochastic chaotic motion. The evolution of the probability density function of the roll response was calculated by solving the stochastic differential equations using the path integral method. It was found that in the probability density function of the system has two peaks for which the response of the system was found to jump from one peak to another for large amplitudes of periodic excitation.Manotov and Naess (2009) developed a combined analytical-numerical approach referred to as the successive-transition method, which is essentially a version of the path-integration solution and is based on an analytical approximation for the transition probability density. The method was applied to onedimensional nonlinear Ito's equation describing the velocity of a ship maneuvering along a straight line under the action of the stochastic drag due to wind or sea waves. It was also used for the problem of ship roll motion up to its possible capsizing. It was indicated that the advantage of the proposed successive-transition is that it provides an account for the damping matrix in the approximation.
The case of Gaussian white noise acting simultaneously with Poisson white noise has not yet been considered in the literature and the present work is an attempt to extend the PI method for this case. In particular, the method will be utilized to examine the ship roll oscillation under parametric normal white noise acting simultaneously with additive Poisson white noise.

\section{PATH INTEGRAL METHOD}

This section provides the general features of the PI method by adopting a simple nonlinear system driven by a white noise described by the one-dimensional equation:

$$
\left\{\begin{array}{l}
\dot{X}(t)=-\alpha X(t)+f(X, t)+W(t) \\
X(0)=X_{0}
\end{array}\right.
$$

where $f(X, t)$ is a deterministic nonlinear function of the response $X(t)$ and time $t, \alpha$ is a positive parameter and $W(t)$ is a white noise and $X_{0}$ is the initial condition that may be either deterministic or random (Gaussian or non-Gaussian).

The starting point of the PI method is the ChapmanKolmogorov equation that holds true, because of the Markovian property of the response:

$$
p_{X}(x, t+\tau)=\int_{D} p_{X}(x, t+\tau \mid \bar{x}, t) p_{X}(\bar{x}, t) d \bar{x}
$$

The numerical implementation of the PI method requires selecting a computational domain $D$. It is convenient to select a symmetrical computational domain with a given maximum size, $x_{\max }=\left|x_{1}\right|$, i.e., $-x_{1} \leq x \leq x_{1}$. The size of the domain is identified by, first, running a Monte Carlo simulation with a low number of samples. Then, dividing the domain in a discrete number of intervals, $n_{x}$, for each grid point, the path integral from equation (2) can be evaluated. One has to evaluate the kernel in equation (2), which requires the conditional joint $p d f$. From the entire set of trajectories of the response process, $X(t)$, one has to select those deterministic values at time $t$, i.e., $\bar{x}$, hereafter denoted as $\bar{X}(\rho)$ (see Fig.1), by solving of the following differential equation:

$$
\left\{\begin{array}{l}
\dot{\bar{X}}(\rho)=-\alpha \bar{X}(\rho)+f(\bar{X}, \rho)+W(t+\rho) \\
\bar{X}(0)=\bar{x}
\end{array}\right.
$$

where $\bar{x}$ is a deterministic initial condition and $0 \leq \rho \leq \tau$. Note that the $c p d f$ of equation (1) coincides with the unconditional $p d f$ of equation (3) evaluated at $\tau$, i.e.,

$$
p_{X}(x, t+\tau \mid \bar{x}, t)=p_{\bar{X}}(x, \tau)
$$

Fig.1 demonstrates the significance of the $c p d f$ of the stochastic process $\bar{X}(\rho)$ evaluated in $\rho=\tau$. These are the general features of the PI method. To this end the problem is 
to specify the kernel, which is dependent on the system and excitation characteristics.

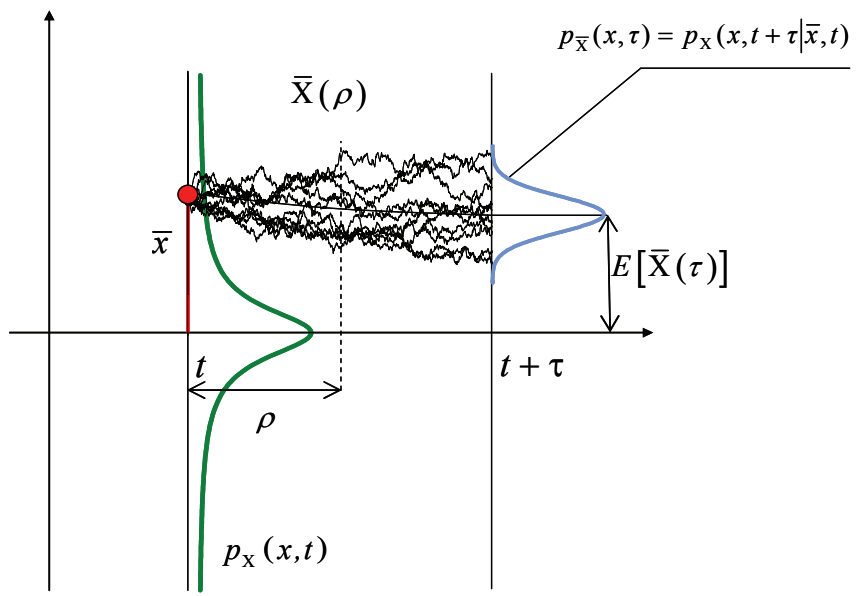

Fig.1Sample functions of the process $\bar{X}(\rho)$ and conditional $p d f$.

\section{Systems under Poisson White Noise}

Sample functions of Poisson white noise process $W_{P}(t)$ in equation (1) may be written in the form

$W_{P}(t)=\sum_{i=1}^{N(t)} R_{i} \delta\left(t-T_{i}\right)$

where $\delta(\bullet)$ is the Dirac's Delta function, $R_{i}$ is the $i$-th realization of the random variable $R$ with assigned probability density function $p_{R}(r) . T_{i}$ is the $i$-th realization of the random variable $T$ independent of $\mathrm{R}$ and distributed in time according to the Poisson law and $N(t)$ is the so-called counting process giving the total number of impulse occurrences in $[0, t)$. The whole process defined by equation (5) is fully described by the cumulants:

$$
K_{n}\left[W_{P}\left(t_{1}\right) W_{P}\left(t_{2}\right) \ldots W_{P}\left(t_{n}\right)\right]=\lambda E\left[R^{n}\right] \delta\left(t_{1}-t_{2}\right) \ldots \delta\left(t_{1}-t_{n}\right)
$$

where $\lambda$ is the mean number of impulse occurrences per unit time.

Replacing $W(t)$ by $W_{\mathrm{P}}(t)$ in equation (1) one can describe the evolution of the system response $p d f$ by the KolmogorovFeller equation

$$
\begin{aligned}
\frac{\partial p_{X}(x, t)}{\partial t} & =-\frac{\partial}{\partial x}\left(\beta(x, t) p_{X}(x, t)\right)-\lambda p_{X}(x, t) \\
& +\lambda \int_{-\infty}^{+\infty} p_{R}(r-y) p_{X}(y) d y
\end{aligned}
$$

where $p_{X}(x, t)$ and $p_{R}(r)$ are the unconditional $p d f$ of the response process $X(t)$ and of the random variable $R$, respectively, and $\beta(x, t)=-\alpha x(t)+f(x, t)$.
The $c p d f p_{X}(x, t+\tau \mid \bar{x}, t)$ may be obtained by evaluating the unconditional $p d f$ in $\tau$ of the following differential equation:

$$
\left\{\begin{array}{l}
\dot{\bar{X}}(\rho)=-\alpha \bar{X}(\rho)+f(\bar{X}, \rho)+W_{P}(t+\rho) ; \quad 0 \leq \rho \leq \tau \\
\bar{X}(0)=\bar{x}
\end{array}\right.
$$

Considering $\tau$ to be small, there are two possible different situations in the interval $(t, t+\tau)$. The first does not contain any spikes and this happens in mean $1-\lambda(t) \tau$ times. The second contains one spike whose amplitude possesses the distribution of $R$. This situation happens $\lambda(t) \tau$ times. Since the time interval $\tau$ is small an approximation of $\bar{X}(t)$, when no spikes occur, is given by solving equation (1), in which we set $W(t)=W_{P}(t)=0$, as:

$$
\bar{X}(\tau)=\bar{x}-\alpha \bar{x} \tau+f(\bar{x}, t) \tau=y(\bar{x}, t, \tau)
$$

The function $(\bar{x}, t, \tau)$ will be denoted as $y(\bar{x})$. When one spike occurs, $\bar{X}(\tau)$ may be evaluated in the approximate form

$$
\bar{X}(\tau)=y(\bar{x})+R
$$

Equation (10) remains valid when $\tau$ is small, but if $\tau$ is not small the location of the spikes within the interval $\tau$ will influence the value $\bar{X}(\tau)$. On the other hand, equation (9) is deterministic over the time increment $1-\ddot{e}(t) \hat{o}$ and thus the entire sample functions of the response process gives a contribution in the $p d f$ of $\bar{X}(\rho)$ in the form $(1-\lambda(t) \tau) \delta(x-y(\bar{x}))$.

In the remaining $\lambda(t) \tau$ sample functions in which a spike occurs one may inspect equation (10), which reveals that $\bar{X}(\tau)$ is a random variable composed of a deterministic plus a random variable with a given $p d f$. It follows that the $p d f$ of $\bar{X}(\rho)$ in is $p_{R}(x-y(\bar{x}))$, and this occurs over the time increment $\ddot{e}(t) \hat{o}$. The resulting $c p d f$ is

$$
\begin{aligned}
p_{X}(x, t+\tau \mid \bar{x}, t)= & (1-\lambda(t) \tau) \delta(x-y(\bar{x})) \\
& +\lambda(t) \tau p_{R}(x-y(\bar{x}))
\end{aligned}
$$

Substituting equation (11) into equation (2), gives

$$
\begin{aligned}
p_{X}(x, t+\tau)= & (1-\lambda(t) \tau) \int_{-\infty}^{+\infty} \delta(x-y(\bar{x})) p_{X}(\bar{x}, t) d \bar{x} \\
& +\lambda(t) \tau \int_{-\infty}^{+\infty} p_{R}(x-y(\bar{x})) p_{X}(\bar{x}, t) d \bar{x}
\end{aligned}
$$

Since $\bar{x}$ and $y(\bar{x})$ are nonlinear the following relationship holds

$$
p_{X}(\bar{x}, t) d \bar{x}=p_{Y}(y(\bar{x}), t) d y(\bar{x})
$$

In this case, equation (12) may be rewritten in the form 


$$
\begin{aligned}
p_{X}(x, t+\tau)= & (1-\lambda(t) \tau) p_{Y}(x, t) \\
& +\lambda(t) \tau \int_{-\infty}^{+\infty} p_{R}(x-y) p_{Y}(y, t) d y
\end{aligned}
$$

where according to equation (9), one can write

$$
p_{Y}(y, t)=p_{X}\left(x=\frac{g(y), t)}{\left|y^{\prime}(x)\right|}\right.
$$

where $g(y)$ is the inverse relationship obtained from equation (9). Equation (14) represents an extension of the PI method to a Poisson white noise input.

Equation (14) is a simple integral equation while the Kolmogorov-Feller equation represented by equation (7) is an integro-differential equation. In order to study the ship roll motion another version of the PI method is necessary for the case of combined normal and Poisson white noise.

\section{Systems under Normal and Poisson white noise excitations}

The $c p d f$ of the response of systems driven simultaneously by Gaussian white noise $W_{0}(t)$ and Poisson white noise $W_{p}(t)$, is not available, and hence the PI method may not be implemented. The normal white noise, $W_{p}(t)$, is characterized by the correlation function:

$$
E\left[W_{0}\left(t_{1}\right) W_{0}\left(t_{2}\right)\right]=q\left(t_{1}\right) \delta\left(t_{1}-t_{2}\right)=q\left(t_{2}\right) \delta\left(t_{1}-t_{2}\right)
$$

where $E[\bullet]$ denotes the ensemble average, $q(t)$ is the strength of the normal white noise (if $W_{0}(t)$ is stationary then $q(t)=q$ ).

Note that we don't have either an equation governing the evolution of response $p d f$ or an analytical expression for the kernel in (2). However, the $c p d f p_{X}(x, t+\tau \mid \bar{x}, t)$ may be obtained by evaluating the unconditional $p d f$ in $\tau$ of the following differential equation:

$$
\left\{\begin{array}{l}
\dot{\bar{X}}(\rho)=-\alpha \bar{X}(\rho)+f(\bar{X}, \rho)+W_{0}(t+\rho)+W_{p}(t+\rho) \\
\bar{X}(0)=\bar{x}
\end{array}\right.
$$

Again considering being small, there are two different situations over the interval $\tau$. The first does not have spikes in the presence of the Gaussian white noise, and this happens over the time increment $1-\lambda(t) \tau$. The second case does have one spike whose amplitude possesses the distribution of $\mathrm{R}$, simultaneously with the Gaussian one. The latter situation happens over the time increment $\lambda(t) \tau$.

For the first case where no spikes occur, one has to solve equation (17) under Gaussian white noise and setting

$W_{p}(t+\rho)=0$. Furthermore, if small, the so called short time Gaussian approximation may be used, i.e., $p_{X}(x, \tau)$ follows a Gaussian distribution. Then in the $p d f$ of whole sample functions of the response may take the following function.

$$
\begin{aligned}
& \left.p_{\bar{X}(\rho)}\right|_{(1-\lambda \tau)}=(1-\lambda \tau) \frac{1}{\sqrt{2 \pi q(t) \tau}} \\
& \quad \times \exp \left(-\frac{(x-\bar{x}-(-\alpha \bar{x}+f(\bar{x}, t)) \tau)^{2}}{2 q(t) \tau}\right)
\end{aligned}
$$

In the remaining time increment $\lambda(t) \tau$ a spike occurs with a Gaussian white noise and one has to consider the $p d f$ of a sum of two random variables one of which is Gaussian and the other is the random variable $\mathrm{R}$ with an assigned $p d f$. It follows that in the whole sample function, in which the spike occurs with the Gaussian white noise, one has a $p d f$ of $\bar{X}(\rho)$ in given by the convolution integral

$$
\begin{aligned}
p_{\left.\bar{X}(\rho)\right|_{\lambda \tau}=} & (\lambda \tau) \int_{-\infty}^{\infty} \frac{1}{\sqrt{2 \pi q(t) \tau}} \\
& \times \exp \left(-\frac{(\xi-\bar{x}-(-\alpha \bar{x}+f(\bar{x}, t)) \tau)^{2}}{2 q(t) \tau}\right) p_{R}(x-\xi) d \xi
\end{aligned}
$$

The resulting $c p d f$ is given by

$$
\begin{aligned}
& p_{X}(x, t+\tau \mid \bar{x}, t)= \\
& (1-\lambda \tau) \frac{1}{\sqrt{2 \pi q(t) \tau}} \exp \left(-\frac{(x-\bar{x}-(-\alpha \bar{x}+f(\bar{x}, t)) \tau)^{2}}{2 q(t) \tau}\right) \\
& +(\lambda \tau) \int_{-\infty}^{\infty} \frac{p_{R}(x-\xi)}{\sqrt{2 \pi q(t) \tau}} \exp \left(-\frac{(\xi-\bar{x}-(-\alpha \bar{x}+f(\bar{x}, t)) \tau)^{2}}{2 q(t) \tau}\right) d \xi
\end{aligned}
$$

Substituting equation (20) into equation (2) yields the PI solution extended to the case of combined case of Gaussian and Poisson white noises. The expression given by equation (20) is applied to the system (17) with $\alpha=1$, and $f(X, t)=-$ $1 / 2 X(t) \mid X(t)$. The Gaussian noise $W_{0}(t)$ is assumed to have unit intensity, $q=1$, while the Poisson noise $W_{p}(t)$ possesses a unitary mean rate arrival $\lambda$, and $p_{R}(x)$ is Gaussian distributed with mean $\mu_{R}=1.5$ and variance $\sigma=2.25$. The initial condition $X_{0}$ is a standard Gaussian distribution. In order to use the short time Gaussian approximation the time interval is selected to be $\tau=0.1$. To validate the proposed method, Monte Carlo (MC) simulation of equation (1) has been performed using Euler's integration scheme with 5000 samples and increment of time $0.005 \mathrm{sec}$. Fig. 2(a) shows a threedimensional representation of the time evolution of the response $p d f$ with initial Gaussian $p d f$.

It is seen that as the time increases the response $p d f$ evolves to a bimodal representation with two peaks around the zero mean value. The results are validated by MC simulation and Fig. 2(b) shows a comparison of the stationary response $p d f$ as generated from $\mathrm{MC}$ simulation and PI solution. The influence of the combined excitations on the response $p d f$ cannot be revealed directly from Fig. 2(b). In order to assess this effect the $p d f$ of each excitation should be separately plotted. 


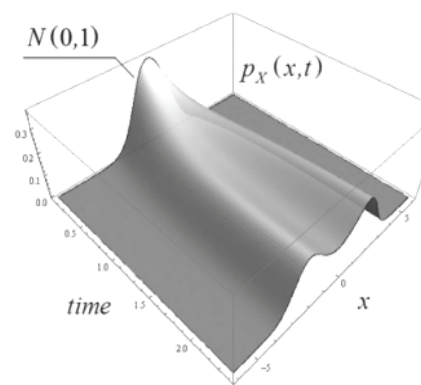

(a)

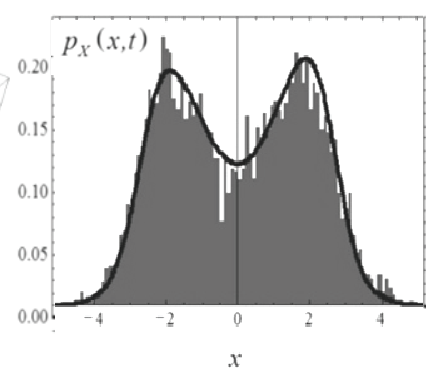

(b)
Fig. 2 Response probability density function: (a) Time evolution of the response $p d f$ as obtained by PI solution; (b) Monte Carlo validation for the stationary state.

\section{SHIP ROLL MOTION UNDER STOCHASTIC AGENCIES}

The study of ship dynamics in following sea waves was first reported by Froude (1863) who observed that ships have undesirable roll characteristics when the frequency of small free oscillations in pitch is twice the frequency of a small free oscillation in roll. It was Paulling and Rosenberg (1959) who formulated the analytical modeling of the coupled roll-pitch motion. This coupled motion is described by two nonlinear differential equations. If the nonlinear effect of roll is neglected, the pitch equation of motion is reduced to a linear differential equation, which is free from roll motion terms. When the pitch equation is solved, its response appears as a coefficient to the restoring moment of roll motion, and the roll equation of motion is reduced to Mathieu's equation. These studies did not take into account the nonlinear effects of drag and restoring moments.

Various models of roll motion containing nonlinear terms in damping and restoring moments have been studied by many researchers and are documented by Ibrahim and Grace (2010). For example, Bass and Haddara (1987, 1988) considered various forms for the roll damping moment and introduced two techniques to identify the parameters of the various models together with a methodology for their evaluation. Taylan (2000) demonstrated that different nonlinear damping and restoring moment formulations reported in the literature have resulted in completely different roll amplitudes, and further yielded different ship stability characteristics. Since ship capsizing is strongly dependent on the magnitude of roll motions, an accurate estimation of roll damping is crucial to the prediction of the ship motion responses. Moreover, the designer should consider the influence of waves on roll damping, especially nonlinear roll damping of large-amplitude roll motion, and subsequently on ship stability.

Different models for the damping moment introduced in the ship rolling equation of motion were proposed in the literature (see, e.g., Dalzell, 1976; Cardo, 1982; Mathisen and Price, 1984). They contain linear-quadratic or linear-cubic in the angular roll velocity. The damping characteristics of a variety of ship shapes and offshore structures undergoing roll oscillation in the presence of ocean waves have been assessed by Chakrabarti (2001). Chakrabarti relied on empirical formulas derived from a series of model experiments reported by Ikeda et al $(1978,1993)$. These experiments were performed on two-dimensional shapes. El-Bassiouny (2007) studied the dynamic behavior of ships roll motion by considering different forms of damping moments consisting of the linear term associated with radiation, viscous damping, and a cubic term due to frictional resistance and eddies behind bilge keels and hard bilge corners. The damping roll moment $B(\phi)$ is nonlinear and may be expressed by the expression, (Chakrabarti, 2001; El-Bassiouny, 2007),

$$
B(\dot{\phi})=c_{1} \dot{\phi}+c_{2} \dot{\phi}|\dot{\phi}|+c_{3} \dot{\phi}^{3}+\ldots=\sum_{k=1}^{K} c_{k} \dot{\phi}|\dot{\phi}|^{k-1}
$$

where $c_{1}$ is the linear damping coefficient and $c_{i}, i=2,3, \ldots$ are the nonlinear damping coefficients. The first term is the usual linear viscous damping; the second is the quadratic damping term originally developed by Morison et al. (1950). It is in phase with the velocity but it is quadratic because the flow is separated and the drag is primarily due to pressure rather than the skin friction. Sarpkaya and Isaacson (1981) provided a critical assessment of Morison's equation, which describes the forces acting on a pile due to the action of progressive waves. The third term is cubic nonlinear damping.

The nonlinear damping moment is proportional to the product of the roll velocity times its absolute value and acts in a direction opposite to the velocity. This is usually represented by the expression, $a \dot{\phi}|\dot{\phi}|$, where $a$ is a constant determined experimentally. The righting arm curve of a ship, known also as the restoring lever has been represented by an odd-order polynomial up to different degrees (Nayfeh and Balachandran, 1995; Arnold, et al., 2004; Surendran, et al., 2005; Bulian, 2005). Different representations of the restoring moment have been proposed in the literature in which the restoring moment $M_{x}(\phi)$ is represented by the general polynomial

$$
M_{x}(\phi)=k_{1} \phi+k_{3} \phi^{3}+k_{5} \phi^{5}+k_{7} \phi^{7}+\ldots
$$

where $k_{1}>0, k_{3}>0, k_{5}>0$, and $k_{7}>0$, for a damaged vessel, but $k_{7}=0$, for an intact vessel.

Based on these considerations the roll dynamics of a ship subjected to simultaneous impulse excitation, $W_{P}(t)$, and parametric random excitation, $W_{0}$, keeping only cubic terms in the restoring moment is described by the equation of motion:

$$
\begin{aligned}
& \ddot{\phi}+2 \tilde{\zeta} \omega_{n} \dot{\phi}+a \dot{\phi}|\dot{\phi}|+\omega_{n}^{2} \phi-\delta \phi^{3}+W_{0}(t) \phi=W_{P}(t) \\
& \phi(0)=\phi_{0} \quad ; \quad \dot{\phi}(0)=\dot{\phi}_{0}
\end{aligned}
$$


where $\phi$ is the ship roll angle, $\omega_{n}$ is the natural frequency of the ship roll oscillation, $\tilde{\zeta}$ is a linear damping factor, the third term represents nonlinear damping, and the cubic term is due to the restoring moment together with the linear term. In particular $W_{0}(t)$ represents the pitch angle which is assumed to be random stationary process and the right-hand side is the sea wave moment acting on the ship and is represented by the Poisson random process given by equation (5). Note that at the roll angle $\phi_{c}= \pm \omega_{n} / \sqrt{\delta}$, the ship experiences capsizing. If we consider $W_{0}(t)$ as a normal white noise, the ship roll dynamics is captured by the solution of a single oscillator under parametric normal white noise acting simultaneously with an external Poisson white noise. Equation (23) may be rewritten in terms of state variables as follows

$$
\dot{\mathbf{Z}}(t)=\mathbf{D Z}(t)+\mathbf{f}(\mathbf{Z}, t)+\mathbf{L} W_{\mathrm{P}}(t)
$$

where $\mathbf{Z}(t)=\left[\begin{array}{c}Z_{1}(t) \\ Z_{2}(t)\end{array}\right]=\left[\begin{array}{c}\varphi(t) \\ \dot{\varphi}(t)\end{array}\right] ; \mathbf{D}=\left[\begin{array}{cc}0 & 1 \\ -\omega_{n}^{2} & -2 \tilde{\zeta} \omega_{n}\end{array}\right]$

$$
\mathbf{f}(\mathbf{Z}(t), t)=\left[\begin{array}{c}
0 \\
-a Z_{2}\left|Z_{2}\right|+\delta Z_{1}^{3}-Z_{1} W_{0}(t)
\end{array}\right] ; \mathbf{L}=\left[\begin{array}{l}
0 \\
1
\end{array}\right]
$$

For this case the Chapman Kolmogorov equation is given in the form:

$$
\begin{aligned}
& p_{Z}\left(z_{1}, z_{2}, t+\tau\right)=\int_{-\infty}^{+\infty} \int_{-\infty}^{+\infty} p_{Z}\left(z_{1}, z_{2}, t+\tau \mid \bar{z}_{1}, \bar{z}_{2}, t\right) \\
& \quad \times p_{Z}\left(\bar{z}_{1}, \bar{z}_{2}, t\right) d \bar{z}_{1} d \bar{z}_{2}
\end{aligned}
$$

The conditional $p d f$ in equation (25) may be derived by considering the $p d f$ of the response over the time interval of the following system

$$
\left\{\begin{array}{l}
\dot{\overline{\mathbf{Z}}}(\rho)=\mathbf{D} \overline{\mathbf{Z}}(\rho)+\mathbf{f}(\overline{\mathbf{Z}}, \rho)+\mathbf{L} W(t+\rho) \\
\overline{\mathbf{Z}}(0)=\overline{\mathbf{Z}}
\end{array}\right.
$$

where the initial conditions are the considered deterministic, $\bar{z}^{T}=\left[\bar{z}_{1}, \bar{z}_{2}\right]$. Equations (26) may be rewritten in the state vector form

$$
\begin{aligned}
& \dot{\bar{Z}}_{1}(\rho)=\bar{Z}_{2}(\rho) \\
& \dot{\bar{Z}}_{2}(\rho)=-2 \tilde{\zeta} \omega_{n} \bar{Z}_{2}(\rho)-\omega_{n}^{2} \bar{Z}_{1}(\rho) \\
& \quad-a \bar{Z}_{2}(\rho)\left|\bar{Z}_{2}(\rho)\right|+\delta \bar{Z}_{1}(\rho)^{3}-\bar{Z}_{1} W_{0}(t+\rho) \\
& \quad+W_{p}(t+\rho) \\
& \bar{Z}_{1}(0)=\bar{z}_{1} ; \bar{Z}_{2}(0)=\bar{z}_{2}
\end{aligned}
$$

For small $\tau$, equations (27) yields the following statistics

$$
\begin{aligned}
& E\left[\bar{Z}_{1}(\tau)\right]=\bar{z}_{1}+\bar{z}_{2} \tau=y_{1}\left(\bar{z}_{1}, \bar{z}_{2}\right) ; \sigma_{\bar{Z}_{1}}^{2}(\tau)=0 \\
& p_{\bar{Z}_{1}}\left(z_{1}, \bar{z}_{1}, \bar{z}_{2} ; \tau\right)=\delta\left(z_{1}-y_{1}\left(\bar{z}_{1}, \bar{z}_{2}\right)\right)(29)
\end{aligned}
$$

Again, for small $\tau$ there are two different situations over time interval $\tau$. The first does not contain spikes in the presence of the normal white noise, and this happens over the time increment $1-\lambda(t) \tau$. The second includes one spike which occurs simultaneously with the normal white noise over the time increment $\lambda(t) \tau$. In the first case we set $W_{P}(t)=0$

$$
\begin{aligned}
& E\left[\bar{Z}_{2}(\tau)\right]=\bar{z}_{2}-\left(2 \tilde{\zeta} \omega_{n} \bar{z}_{2}+\omega_{n}^{2} \bar{z}_{1}+a \bar{z}_{2}\left|\bar{z}_{2}\right|-\delta \bar{z}_{1}^{3}\right) \tau \\
& \quad=y_{2}\left(\bar{z}_{1}, \bar{z}_{2}\right) \\
& \sigma_{\bar{z}_{2}}^{2}(\tau)=E\left[\bar{Z}_{2}^{2}(\tau)\right]-\left(E\left[\bar{Z}_{2}(\tau)\right]\right)^{2}=\bar{z}_{1}^{2} q \tau
\end{aligned}
$$

Since $Z_{1}$ is deterministic, the two processes $Z_{1}$ and $Z_{2}$ are independent. In the absence of spikes the contribution for the whole conditional $p d f$ in equation (25) is given in the form

$$
\begin{aligned}
& p_{Z}\left(z_{1}, z_{2}, t+\tau \mid \bar{z}_{1}, \bar{z}_{2}, t\right)_{\text {no spikes }} \\
& =(1-\lambda \tau) \frac{\delta\left(z_{1}-\left(\bar{z}_{1}+\bar{z}_{2} \tau\right)\right)}{\sqrt{2 \pi \bar{z}_{1}^{2} q \tau}} \times \exp \left(-\frac{\left(z_{2}-y_{2}\left(\bar{z}_{1}, \bar{z}_{2}\right)\right)^{2}}{2 \bar{z}_{1}^{2} q \tau}\right)
\end{aligned}
$$

The other contribution is given by the convolution integral of both excitations:

$$
\begin{aligned}
& p_{Z}\left(z_{1}, z_{2}, t+\tau \mid \bar{z}_{1}, \bar{z}_{2}, t\right)_{\text {one spike }} \\
= & \frac{\lambda \tau \delta\left(z_{1}-\left(\bar{z}_{1}+\bar{z}_{2} \tau\right)\right)}{\sqrt{2 \pi \bar{z}_{1}^{2} q \tau}} \\
& \times \int_{-\infty}^{+\infty} \exp \left(-\frac{\left(\xi-y_{2}\left(\bar{z}_{1}, \bar{z}_{2}\right)\right)^{2}}{2 \bar{z}_{1}^{2} q \tau}\right) p_{R}\left(z_{2}-\xi\right) d \xi
\end{aligned}
$$

The $c p d f$ of the kernel of equation (25) is

$$
\begin{aligned}
& p_{Z}\left(z_{1}, z_{2}, t+\tau \mid \bar{z}_{1}, \bar{z}_{2}, t\right) \\
& =\delta\left(z_{1}-\left(\bar{z}_{1}+\bar{z}_{2} \tau\right)\right) \\
& \times\left[\frac{(1-\lambda \tau)}{\sqrt{2 \pi \bar{z}_{1}^{2} q \tau}} \exp \left(-\frac{\left(z_{2}-y_{2}\left(\bar{z}_{1}, \bar{z}_{2}\right)\right)^{2}}{2 \bar{z}_{1}^{2} q \tau}\right)\right. \\
& \left.+\frac{\lambda \tau}{\sqrt{2 \pi \bar{z}_{1}^{2} q \tau}} \int_{-\infty}^{+\infty} \exp \left(-\frac{\left(\xi-y_{2}\left(\bar{z}_{1}, \bar{z}_{2}\right)\right)^{2}}{2 \bar{z}_{1}^{2} q \tau}\right) p_{R}\left(z_{2}-\xi\right) d \xi\right]
\end{aligned}
$$

The ship roll response $p d f$ is determined for $\zeta=0.1, \omega=1$, $a=0.005$, and $\delta=0.006$. The external Poisson white noise $W_{P}(t)$ has a jump Gaussian distribution $p_{R}(x)$ with zero mean and standard deviation $\sigma_{R}=0.07$, and the mean rate arrival 
$\lambda=1 / 3$. The parametric normal white noise $W_{0}(t)$ is assumed to possess an intensity parameter $q=0.05$. The initial condition is $p_{\phi \dot{\phi}}(\phi, \dot{\phi} ; 0)$ assumed to be a bivariate normal distribution with vector mean $(0.05,0.025)$ and covariance matrix $\Sigma=\left(\begin{array}{cc}0.133^{2} & 0 \\ 0 & 0.133^{2}\end{array}\right)$.

Integrals have been performed numerically on a grid of $\Delta \phi=\Delta \phi=0.01$ and time step $\tau=0.1 \mathrm{sec}$.

Fig. 3 shows the evolution of the response $p d f$ at various time instants starting from the initial condition. It is seen that the ship response $p d f$ is essentially non-Gaussian and the mean value is shifted with time towards the zero value. The observed asymmetry of the response $p d f$ is mainly attributed to the system nonlinearity and to the fact that the excitation is due to the beam sea waves (i.e., only of one side of the ship beam).

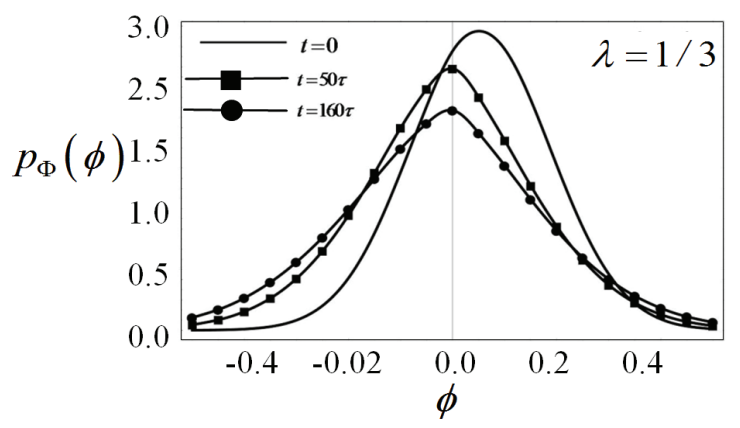

Fig. 3 Response $p d f$ at discrete time intervals.

The influence of the mean rate arrival is evaluated by considering different values of $\lambda=1 / 3,1$, and 3 . These values are selected with large difference to reveal the influence the mean rate on the response $p d f$ as it increases. The response $p d f$ of ship roll angle is plotted in Fig. 4 and reveals that as the mean rate arrival of ice impacts increases the response $p d f$ is found to be spread and the extreme ship roll angle is extended towards a dangerous value of the capsizing roll angle. Furthermore, as the mean rate arrival increases, the peak of the response $p d f$ is reduced. In other words, the probability of the ship response to reach the capsizing angle increases as the mean rate arrival increases.

\section{CONCLUSIONS}

The method of path integral has been implemented to the problem of ship roll oscillations subjected to simultaneous white noise parametric excitation and addition pulse excitation. The additive excitation simulates the pulse of random events of floating ice impact to the ship. It has been found that the evolution of the ship response probability density function is essentially non-Gaussian and the mean value is shifted with time towards a zero value. As the mean rate arrival of ice impacts increases the response $p d f$ is found to be spread and the extreme ship roll angle is extended towards a dangerous value of the capsizing roll angle.
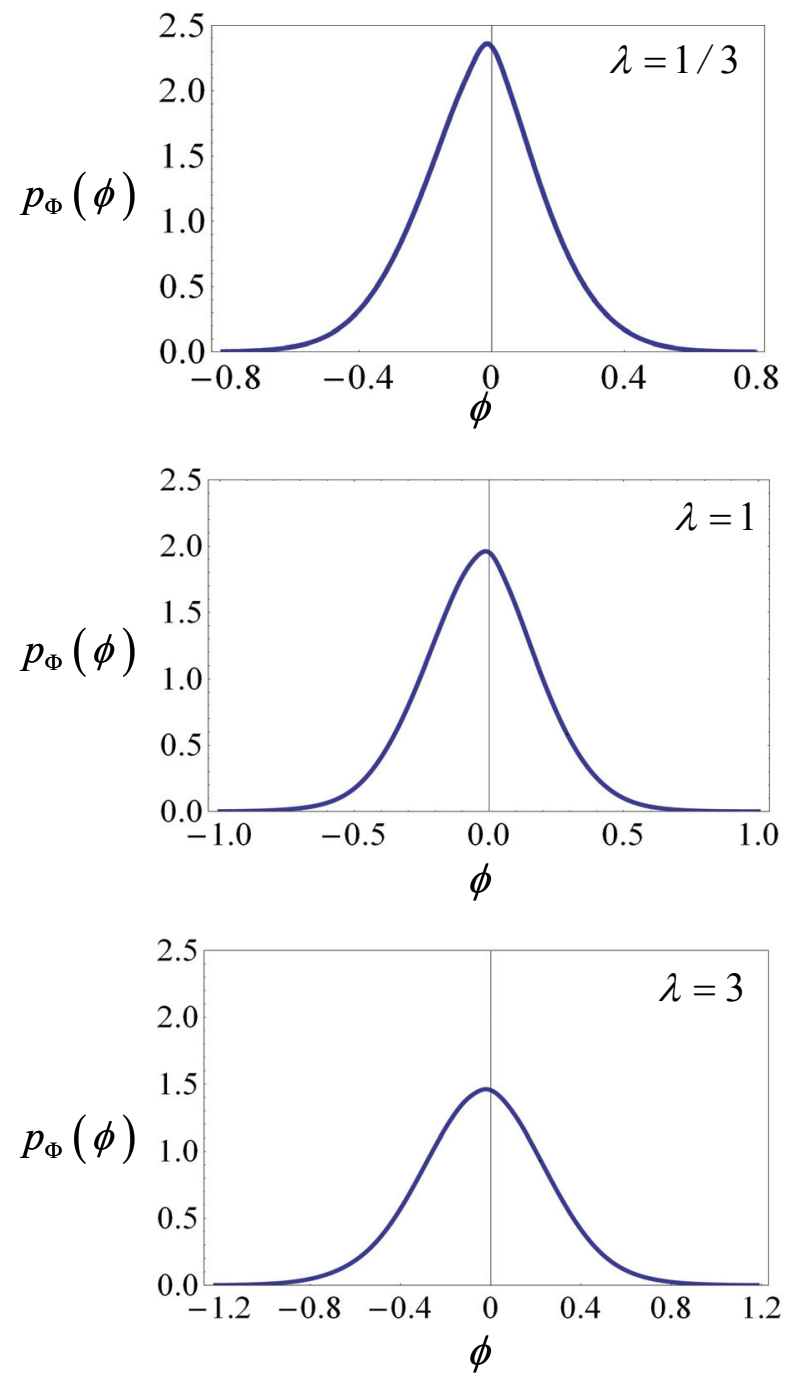

Fig. 4 Response $p d f$ of ship roll angle for three different values of the mean rate arrival. Parameter $\lambda=1 / 3,1$ and. 3 .

\section{ACKNOWLEDGEMENT}

R. A. Ibrahim is supported by a grant from the Office of Naval Research under Award No: N00014-05-1-0040. Dr. Kelly B. Cooper is the Program Director. The authors would like to express their appreciation to Professor P. Spanos for fruitful comments and private discussions.

\section{REFERENCES}

Arnold, L. Chueshov, I. and Ochs, G., 2004. Stability and Capsizing of Ships in Random Sea - A Survey. Nonlin Dyn, 36, pp.135-179. 
Atkinson, J.D., 1973. Eigenfunction expansions for randomly excited non-linear systems. J Sound Vib, 30, pp. 153-172.

Barone, G. Navarra G. and Pirrotta, A., 2008. Probabilistic response of linear structures equipped with nonlinear damper devices (PIS method). Probab Eng Mech, 23(23), pp.125-133.

Bass, D.W. and Haddara, M.R., 1987. On the modeling of the nonlinear damping moment for the rolling motion of a ship. Proc Int Assoc Sci Techn (IASTED), Paris, France, pp. 346-349.

Bass, D.W. and Haddara, M.R., 1988. Nonlinear models of ship roll damping. Int Shipbuilding Prog, 35(401), pp. 5-24.

Bulian, G., 2005. Nonlinear parametric rolling in regular waves - a general procedure for the analytical approximation of the GZ curve and its use in time domain simulations. Ocean Eng, 32, pp. 309-330.

Cardo, A., 1982. On damping models in free and forced rolling motion. Ocean Eng, 9 (2), pp. 171-179.

Caughey, T.K. and Dienes, J.K., 1961. Analysis of Nonlinear First-Order system with a White Noise Input. $J \mathrm{Appl}$ Phys 32(11), pp. 2476-2483.

Chakrabarti, S., 2001. Empirical calculations of roll damping for ships and barges. Ocean Eng, 28(7), 915-932.

Dalzell, J.F., 1976. A note on the form of ship roll damping. J Ship Res 22, pp. 178-185.

Dimentberg, M.F., 1982. An Exact Solution to a Certain Nonlinear Random Vibration Problem. Int $J$ Nonlin Mech, 17(4), pp. 231-236.

Di Paola, M. and Pirrotta, A., 1999. Nonlinear systems under impulsive parametric input. Int J Nonlin Mech, 34, pp. 843-851.

Di Paola, M. and Santoro, R., 2008. Nonlinear systems under Poisson white noise handled by path integral solution. $J$ Vib Control, 14(1-2), pp. 35-49.

El-Bassiouny, A.F., 2007. Nonlinear analysis for a ship with a general roll-damping model. Physica Scripta, 75, pp. 691-701.

Froude W.F., 1863. Remarks on Mr. Scott Russell's paper on rolling. Trans Inst Naval Res, 4, pp. 232-275.

$\mathrm{Gu}$, J.Y., 2006. Calculation of ship rolling probability using a new path integration method. $J$ Ship Mech, 10(6), pp. 43-52.

Ibrahim, R.A. Chalhoub, N.G. and Falzarano, J., 2007. Ice Interaction with Ships and Ocean Structures and Their Control. ASME, Appl Mech Rev, 60(5), pp. 246-289.

Ibrahim, R.A. and Grace, I.M., 2010. Modeling of ship roll dynamics and its coupling with heave and pitch. Mathematical Problems in Engineering, pp. 1-31.

Ikeda, Y. Himeno, Y. and Tanaka, N., 1978. Components of Roll Damping of Ships at Forward Speed,Journal of Society Naval Architects, 143, pp. 121-133.

Ikeda, Y. Fujiwara, T. and Katayama, T., 1993. Roll damping of a sharp-cornered barge and roll control by a new-type stabilizer. Proc 3rd Int Offshore and Polar Engineering Conference, Singapore, 6-11 June 1993, pp. 634-539.
Köylüoğlu, H.U. Nielsen, S.R.K. and Iwankiewicz, R., 1995. Response and reliability of Poisson-driven systems by path integration. ASCE, J Eng Mech, 121(1), pp. 117130.

Kwon, S.H. Kim, D.W. and Chung, J., 1993. Application of path integral solution to ship rolling motion. Proc $3 r d$ Int Offshore and Polar Engineering Conference, Singapore, 6-11 June 1993, pp. 657-660.

Lin, H. and Yim, S.C.S., 1995. Chaotic roll motion and capsize of ships under periodic excitation with random noise. Appl Ocean Res, 17(3), pp. 185-204.

Liqin, L. and Yougang, T., 2007. Stability of ships with water on deck in random beam waves. J Vibr Control, 13(3), pp. 269-280.

Mamontov, E. and Naess, A., 2009. A'n analytical-numerical method for fast evaluation of probability densities for transient solutions of nonlinear Ito's stochastic differential equations. Int J Engrg Sci, 47(1), pp. 116130.

Mathisen, J.B. and Price, W.G., 1984. Estimation of ship roll damping coefficients. Trans, RINA, 127, pp. 295-307.

Meyerhoff, W.K. and Schlachter, G., 1980. An approach for the determination of hull-girder loads in a seaway including hydrodynamic impacts. Ocean Eng, 7(2), pp. 305-326.

Morison, J.R. O'Brien, M.P. Johnson, J.W. and Schaaf, S.A., 1950. The force exerted by surface waves on a pile. AIME, 189, pp.149-154.

Nayfeh, A.H. and Balachandran, B., 1995. Applied Nonlinear Dynamics. Wiley Series in Nonlinear Science, New York.

Paulling, J.R. and Rosenberg, R.M., 1959. On unstable ship motions resulting from nonlinear coupling. J Ship Res, 2, pp. 36-46.

Proppe, C., 2003. Exact stationary probability density functions for nonlinear systems under Poisson white noise excitation. Int $J$ Nonlin Mech, 38, pp. 557-564.

Sarpkaya, T. and Isaacson, M., 1981. Mechanics of Wave Forces on Offshore Structures. Van Nostrand Reinhold, New York.

Spencer, B.F. and Bergman, L.A., 1993. On the numerical solution of the Fokker-Planck equation for nonlinear stochastic systems. Nonlin Dyn, 4, pp. 357-372.

Surendran, S. Lee, S.K. Reddy, J.V.R. and Lee, G., 2005. Nonlinear roll Dynamics of a Ro-Ro Ship in Waves. Ocean Eng, 32, pp. 1818-1828.

Taylan, M., 2000. The effect of nonlinear damping and restoring in ship rolling. Ocean Eng, 27, pp. 921-932.

Timco, G. W. and Johnston, M., 2004. Ice loads on the caisson structures in the Canadian Beaufort Sea. Cold Reg Sci Techn, 38, pp. 185-209.

Vasta, M., 1995. Exact stationary solution for a class of nonlinear systems driven by a non-normal delta-correlated processes. Int J Nonlin Mech, 30(4), pp. 407-418. 